\title{
Conhecimento na clínica em saúde: da produção à utilização
}

\author{
Cristina Lavareda Baixinho ${ }^{1}$, Óscar Ferreira², Fátima Mendes \\ Marques ${ }^{1}$, Maria Helena Presado ${ }^{3}$, Mário Cardoso ${ }^{3}$, Armando David \\ Sousa ${ }^{4}$ y Tiago Nascimento ${ }^{2}$ \\ ${ }^{1}$ Departamento de Enfermagem de Reabilitação, Escola Superior de Enfermagem de \\ Lisboa, Portugal | crbaixinho@esel.pt | https://orcid.org/0000-0001-7417-1732 | \\ fmarques@esel.pt |https://orcid.org/ 0000-0001-6581-6711 \\ ${ }^{2}$ Departamento de Fundamentos de Enfermagem, Escola Superior de Enfermagem de \\ Lisboa, Portugal | oferreira@esel.pt | https://orcid.org/0000-0002-1703-347X | \\ tnascimento@esel.pt | https://orcid.org/0000-0003-3646-9057 \\ ${ }^{3}$ Departamento de Saúde Materna e Obstétrica, Escola Superior de Enfermagem de Lisboa, \\ Portugal | mhpresado@esel.pt |https://orcid.org/ 0000-0002-6852-7875 | \\ mmcardoso@esel.pt | https://orcid.org/0000-0003-4961-9026 \\ ${ }^{4}$ Centro Hospitalar do Funchal, Madeira, Portugal | armandodav@gmail.com | \\ https://orcid.org/ 0000-0002-6393-5896
}

\begin{abstract}
Resumo: A produção de investigação e a utilização dos seus resultados não têm evoluído da mesma forma. O crescimento exponencial de estudos na área da saúde tem possibilitado o aumento da evidência para sustentar uma prática clínica de qualidade e segura. A revisão da literatura e a experiência do grupo de investigadores demonstram que a transferência do conhecimento para a clínica é mais lenta, com implicações na práxis e impacto negativo nos sistemas de saúde e nos custos associados. O grupo tem trabalhado aspetos relacionados com a transferência do conhecimento para a prática clínica e advoga que, desde o desenho do projeto de investigação à obtenção dos achados, importa prever a utilização do novo conhecimento. Evidenciar os melhores resultados e indicadores de saúde, através de cuidados centrados na pessoa, pode influenciar as políticas e os decisores políticos, contribuindo para um sistema mais equitativo e sustentável, com participação e envolvimento dos profissionais.
\end{abstract}

Palavras Chave: Saúde; Enfermagem; Prática Clínica; Investigação Qualitativa; Transferência de Conhecimento.

\section{Introdução}

As ciências da área da saúde têm assistido nas últimas três décadas ao aumento exponencial da produção de conhecimento. Apesar do incremento da produtividade os investigadores observam que a introdução dos resultados da pesquisa na clínica continua a ser lenta, apontando como justificação para esta dificuldade a socialização profissional, os modelos da Prática Baseada na Evidência (PBE) e a predominância da valorização dos resultados da investigação inserida num paradigma quantitativo (Baixinho, Ferreira, Marques, Presado, Cardoso, \& Sousa, 2018), o que pode conduzir ao desuso do conhecimento na clínica em saúde. De salientar que a PBE é uma preocupação dos contextos da práxis, mas a tónica tem sido no uso de modelos lineares e unidirecionais, para levar passivamente a informação dos investigadores aos utilizadores (profissionais da prática clínica) (Baumbush et al., 2008), o que não garante a efetiva apropriação e utilização dos resultados da pesquisa.

Pearson, Jordan e Munn (2012) advogam que a opção pelos ditos modelos tradicionais de PBE traduzem-se em dificuldades que, os autores, classificam em: a) dificuldades em identificar as necessidades da saúde globais para a descoberta de novos conhecimentos, por meio da pesquisa; b) ausência de pontes entre esse novo conhecimento e a produção de pesquisa clínica e social para garantir a sua aplicabilidade e c) dificuldades na incorporação do conhecimento produzido em boas práticas e em políticas do sistema de saúde. 
Pereira (2013) reforça esta visão ao referir que o acesso aos resultados da investigação e a introdução dos resultados dos estudos na prática clínica demoram algum tempo e, em alguns casos, os resultados da pesquisa não são integrados efetivamente nos locais de prestação de cuidados a que se destinam, onde poderiam gerar uma melhoria no cuidado de saúde.

Alguns autores referem que os obstáculos a uma PBE podem ter origem na própria formação pré-graduada nos cursos da área da saúde. No caso específico da enfermagem, a introdução de educação em PBE nos currículos em enfermagem é fortemente recomendada (Mena-Tudela, González-Chordá, Cervera-Gasch, Maciá-Soler, \& OrtsCortés, 2018; Baixinho et al., 2019), tanto pela inclusão destes conteúdos na unidade curricular de investigação, como também, pela introdução dos estudos e discussão da sua evidência nas diferentes disciplinas específicas de enfermagem.

Alguns investigadores vão mais longe e defendem que a melhoria dos conhecimentos, atitudes e competências dos estudantes ao nível da evidência científica é maior quando a aprendizagem é integrada no estágio clínico (Mena-Tudela et al., 2018), mas a este nível também há adversidades, associadas à falta de tempo, ao deficiente suporte organizacional e até lacunas nas capacidades para pesquisar, criticar e sintetizar a literatura como barreiras à incorporação da PBE, provavelmente por os profissionais de saúde não terem sido educados no paradigma da PBE (Mena-Tudela et al., 2018).

Aprender a investigar é um processo complexo que exige conhecimentos sobre métodos e técnicas e implica o desenvolvimento de competências de síntese, intercâmbio e aplicação do conhecimento. Como referem Albarqouni et al. (2018) o desenvolvimento de competências científicas implica a aquisição e o desenvolvimento de um conjunto mínimo essencial de atributos, conhecimentos, habilidades e atitudes, que permitem ao profissional realizar atividades baseadas na evidência. Estas dificuldades/obstáculos levam alguns investigadores a defender que os modelos tradicionais de transferência e/ou acesso ao conhecimento não são eficazes, pugnando pela existência de modelos mais interativos e produtivos (Baumbush et al., 2008), que possam atuar não só ao nível individual, mas também organizacional das políticas de saúde, garantindo que, desde 0 início, a pesquisa é projetada para estar atenta às necessidades da prática (CIHR, 2014). Convém referir que a cultura organizacional, o tempo necessário à prestação direta de cuidados e a gestão diária de outras tarefas, dificultam a busca e discussão da evidência, tornando-se obstáculos à PBE.

Acrescem as adversidades em transferir o conhecimento (TC) obtido por estudos de natureza qualitativa (Baixinho et al., 2018; Baixinho et al., 2019), essenciais para a compreensão das práticas, comportamentos, experiências e decisões do indivíduo e sua família sobre o seu projeto de saúde, inserido no seu projeto de vida (Baixinho et al., 2018).

Face ao exposto, são objetivos deste capítulo: a) Descrever e analisar a experiência do grupo de investigadores no âmbito do projeto Transição Segura e b) Debater a 'evidência qualitativa' e a elaboração de produtos/instrumentos que auxiliam na incorporação dos resultados de estudos de natureza qualitativa e mista na clínica.

\section{A pesquisa / método / o projecto}

O projeto que divulgamos neste Handbook, denominado transição Segura, é um projeto colaborativo e participativo entre academia e instituições de saúde (um hospital e um agrupamento de centros de saúde), que tem como finalidade a utilização da evidência na clínica, com envolvimento de todos os parceiros, suportados por uma metodologia de trabalho que permite gerar mudanças duradouras e conhecimento "útil", motivar profissionais, melhorar o marketing das instituições, diminuir custos e acima de tudo permitir o melhor cuidado, no momento adequado, com o conhecimento atual, e com traduções diretas na melhoria da saúde da população, na acessibilidade aos serviços e diminuição dos custos (Baixinho et al., 2017). 
O efetivo uso do conhecimento implica seguir um conjunto de passos que garanta desde o desenho de estudo à transferência dos resultados para a clínica (Baixinho et al., 2019).

A definição atempada do como e do quê para agilizar a TC, obtidos através de achados predominantemente qualitativos é um desafio na saúde onde predominam as guidelines, os ensaios randomizados e as revisões sistemáticas com meta-análise (Baixinho et al., 2018). Matheus (2009) observa que o aumento das pesquisas qualitativas, tem trazido conhecimento novo, que está disperso, motivo pelo qual têm pouco impacto na prática, sendo subutilizado na formulação de políticas públicas de saúde.

Por isso, o autor recomenda a elaboração de meta-sínteses para transformar vários estudos qualitativos num novo estudo (Matheus, 2009). E, apesar de concordarmos com esta orientação, parece-nos que o desafio é mais complexo porque o resultado deste estudo secundário de base qualitativa, tal como acontece com os de meta-análise, tem de chegar aos contextos com recurso a modelos interativos de PBE e com a utilização de estratégias e produtos que facilitem a incorporação da evidência nos cuidados de saúde.Nos subpontos seguintes apresentamos as fases da evolução do projeto, discutimos a sistematização e a evidência dos estudos qualitativos para possibilitar a sua introdução nos contextos de prática clínica.

\subsection{Fases do processo}

O projeto transição segura tem apostado no desenvolvimento de novos modelos de interação academia-práxis, para possibilitar a intervenção clínica sustentada na evidência e aumentar a adesão dos profissionais a comportamentos, atitudes de literacia científica, com capacidade de análise e crítica face à investigação e a sua utilização segura.

Desde 2014 que um grupo de profissionais das instituições parceiras e estudantes do Curso de Licenciatura em Enfermagem (CLE), têm sido envolvidos em atividades de investigação, de acordo com as necessidades dos projetos que decorrem nos diferentes serviços de internamento hospitalar.

Convém salvaguardar que os projetos são díspares em termos da sua natureza, da metodologia utilizada e da fase em que se encontram, por isso as atividades que se desenrolam num projeto podem não corresponder à de outro porque os objetivos e são diferentes, bem como o próprio cronograma.

As atividades em que profissionais e estudantes estão envolvidos (Figura 1) podem estar associadas à definição de problemas que necessitam de resolução através de investigação (primária ou introdução da evidência); definição de prioridades; desenho de estudos; síntese do conhecimento; uso (ou aplicação prática dos resultados da investigação) e avaliação da aceitação, pelos utilizadores, seguindo as indicações do Instituto Canadiano de Investigação em Saúde (CIHR, 2014).

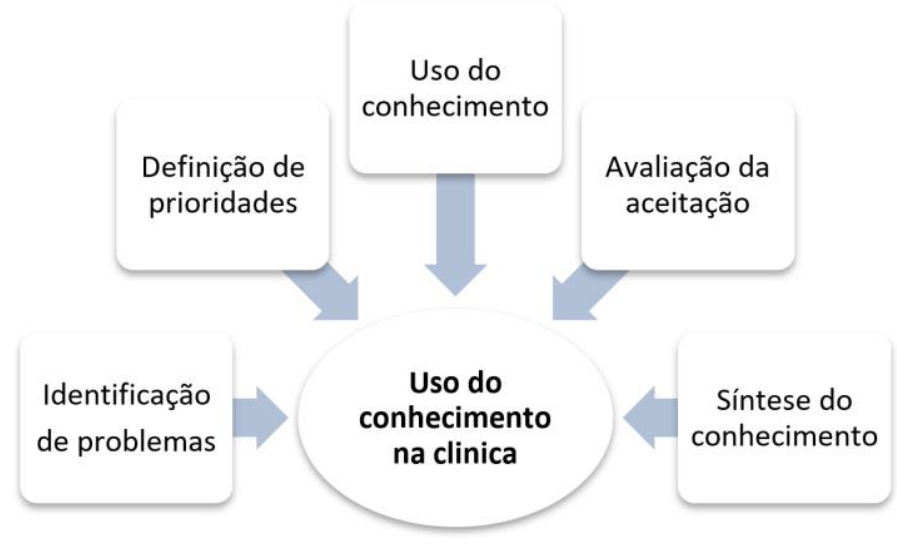

Fig. 1 - Atividades colaborativas de investigação 
Em todos os projetos, independentemente do seu estádio, há a preocupação de integrar no planeamento transferência do conhecimento, para o ambiente específico onde decorre a prática; com a definição de um conjunto de estratégias que promovam a adesão à evidência e resultados relacionados com a saúde e outros e simultaneamente garantir a avaliação e monitorização regular do modelo utilizado para determinar a sua aceitação e avaliar indicadores.

A questão da monitorização do envolvimento dos diferentes autores justifica-se em qualquer fase e/ou atividade para se conseguir estruturar o processo e para apoiar os profissionais e estudantes, provavelmente menos hábeis na arte de investigar, a compreensão da utilidade das mesmas e o impacto que podem ter na melhoria da prestação de cuidados.

Concordamos que para (re)pensar a transferência do conhecimento para a clínica o investigador tem de questionar os modos de investigar e a forma como os profissionais da clínica compreendem a investigação, não só os resultados, como todo o processo, dado que a compreensão alargada de como foi garantida a validade, adequação e objetividade dos achados, permite uma apreciação da qualidade dos mesmos (Baixinho et al., 2018).

Enquanto equipa estamos numa fase de discutir a introdução da evidência qualitativa nos contextos clínicos, pela utilização de revisões da literatura com metodologia científica e produção de produtos para facilitar a transferência do conhecimento.

\subsection{Instrumentos}

Um dos instrumentos utilizados tradicionalmente na PBE tem sido as revisões da literatura com metodologia científica. As revisões de literatura têm caminhado ao longo dos anos com um aumento da complexidade de metodologia, possibilitando uma maior adequação às necessidades académicas e permitindo um maior contributo para uma efetiva e credível PBE e consequente TC.

A sistematização destas revisões, como mecanismo para agregar e organizar um maior número de dados e informação, tem permitido divulgar os resultados das pesquisas primárias, cujo aumento exponencial em algumas áreas dificulta a sua divulgação junto dos profissionais de saúde.

Uma PBE desenvolve-se a partir do pressuposto da aquisição do conhecimento mais atual, com o objetivo de aumentar os níveis de eficácia e eficiência na práxis, pelo uso consciente, explícito e judicioso da investigação para dar resposta a um fenómeno identificado na prática.

Uma revisão sistemática, resume os resultados de estudos de saúde disponíveis cuidadosamente projetados e fornece um alto nível de evidência sobre a eficácia das intervenções de saúde (Cochrane, 2018). Desta forma, é possível analisar e reunir consensos, realizando juízos e informando acerca dos cuidados a prestar em um futuro. As revisões sistemáticas envolvem vários passos dos quais se destacam o identificar da questão de investigação (recorrendo, por exemplo, à ferramenta PICO), a definição de critérios de inclusão e exclusão, a pesquisa dos estudos, a seleção dos estudos que se enquadram nos critérios anteriormente previstos, a extração dos dados obtidos, a análise de vieses nos estudos selecionados, a apresentação dos resultados e finalmente, a disseminação desses resultados (Choi, Cheng, \& Greenberg, 2019).

A reflexão do grupo de investigação visa a sistematização da evidência qualitativa para a introdução dos achados na clínica, bem como, o tipo de articulação entre os investigadores e as 'equipas do terreno'. A este nível as revisões da literatura com método científico, nomeadamente as meta-sínteses, os meta-sumários, as revisões de estudos mistos (que permitem combinar estudos quantitativos com qualitativos ou então resultados com estudo de processo), as revisões integrativas da literatura (RIL) e a revisão scoping são instrumentos importantes para numa primeira fase organizar os resultados dos estudos primários e numa segunda fase produzir produtos para a TC. 
Não sendo nosso intuito explorar os diferentes tipos de revisão da literatura ressalvamos que, em relação às meta-sínteses e às RIL, tal como as revisões sistemáticas permitem uma análise de um fenómeno em específico. Também estas, permitem uma análise adequada da situação, porque oferecem um equilíbrio apropriado entre uma estrutura objetiva, uma abordagem rigorosamente científica da análise dos dados e a contribuição necessária da subjetividade do pesquisador na construção do trabalho final (Lachal, Revah-Levy, Orri, \& Moro, 2017; Mendes, Silveira, \& Galvão, 2008). Como em qualquer outro estudo primário, a revisão sistemática permite uma avaliação rigorosa, imparcial e abrangente da literatura. Uma revisão mal conduzida pode induzir em erro, a estratégia de pesquisa é um dos determinantes da qualidade da revisão sistemática, não se podendo produzir uma boa revisão baseada numa fraca estratégia de pesquisa. A revisão sistemática, comparada com a investigação primária, requer relativamente poucos recursos, permitindo que os profissionais normalmente não envolvidos em investigação produzam artigos clinicamente relevantes e de alta qualidade (Donato, 2019).

Este tipo de revisões requer análises cuidadosas dos paradigmas de pesquisa utilizados, de acordo com as suposições sobre a forma como o conhecimento é gerado e o que constitui efetivamente conhecimento válido (Mendes, Silveira, \& Galvão, 2008; Urquhart, 2010) e passível de ser usado em contextos com características singulares. As metasínteses desenrolam-se de acordo com a formulação da questão, a definição de fontes e respetivo protocolo de pesquisa, a seleção dos estudos, a respetiva avaliação e tradução, finalizando com a síntese dos achados (Atkins, Lewin, Smith, Engel, Fretheim, \& Volmink, 2008).

Ainda no que respeita às meta-sínteses enquanto metodologia qualitativa, é possível desenvolver previamente um meta-sumário, como caminho para a meta-síntese. Falamos assim de uma agregação de resultados quantitativos que são resumos temáticos ou linhas orientadoras de pesquisa (Martsolf, Draucker, Cook, Ross, Stidham, \& Mweemba, 2010). Esta metodologia inclui a extração, o agrupamento e a formatação dos achados e o cálculo dos tamanhos dos efeitos de frequência e intensidade, e que pode ser usada para produzir sínteses de pesquisa mistas e realizar análises a posteriori da relação entre relatórios e achados. Os resultados com maior frequência são utilizados com objetivo de encontrar evidências que demonstrem a repetição da validade na pesquisa quantitativa através da descoberta de um padrão ou tema (Sandelowksi, Barroso, \& Voils, 2007; Hestevik, Molin, Debesay, Bergland, \& Bye, 2019).

Este tipo de recursos obriga por parte dos investigadores e/ou profissionais, a uma formação adequada e exigente, de forma a entender os protocolos subjacentes às várias tomadas de decisão.

\subsection{Participantes}

Como referido anteriormente participam neste projeto professores, estudantes e investigadores da Escola Superior de Enfermagem de Lisboa e profissionais de saúde das instituições parceiras, que firmaram um protocolo de cooperação para o desenvolvimento do projeto. A Comissão de Ética do Hospital autorizou a realização dos estudos em curso (parecer de aprovação 09/2019).

Os profissionais de saúde do hospital foram escolhidos pelos gestores do serviço em função da sua formação académica, capacidade de liderança e funções exercida nos diferentes serviços de internamento hospitalar (medicinas, ortopedia, unidade de cuidados intensivos, serviço de urgência, pediatria, ginecologia e obstetrícia, psiquiatria).

Os critérios de seleção dos profissionais dos agrupamentos de Centros de Saúde foram: coordenarem as unidades de cuidados na comunidade e/ou prestarem cuidados às pessoas após a sua saída de um internamento hospitalar.

Em relação aos estudantes de enfermagem, têm sido envolvidos os estudantes do $4^{\circ}$ ano, 2 을 semestre que fazem o seu estágio de integração à vida profissional nos serviços das instituições parceiras. 
A opção por estudantes finalistas deve-se à complexidade das atividades, exigindo conhecimento sobre paradigmas, métodos e técnicas de investigação, competências na pesquisa em bases de dados, bem como de maturidade necessária para este tipo de articulação com os professores e as 'equipas do terreno'.

De salientar que o desenvolvimento de competências acrescidas e a mudança do papel dos profissionais da prática como coprodutores do conhecimento, tem incrementado um compromisso contínuo e duradouro com os projetos de investigação nas suas áreas clínicas (Baixinho et al., 2017; Baixinho et al., 2018).

\subsection{Análise de dados}

Como explicitado anteriormente temos a preocupação da sistematização da evidência e a construção de produtos para facilitar a transferência do conhecimento.

A etapa seguinte à identificação da evidência, ou nos serviços onde decorrem projetos de investigação-ação-formação, após a análise dos achados a preocupação, é a TC para a clínica com trabalho conjunto com os profissionais envolvidos no projeto e a criação de produtos que promovam a tomada de decisão clínica baseada nos resultados de investigação. A disparidade entre os resultados de investigação e a prática clínica é frequentemente referida como uma lacuna. O desconhecimento de instrumentos de pesquisa e a interpretação dos resultados da investigação disseminada são consideradas das principais barreiras à transferência do conhecimento (Lage et al., 2016).

Este processo de TC necessita de conhecimento sobre a construção da evidência, combinado com a perícia individual na interpretação e análise dos resultados, bem como na tomada de decisão do método/técnicas a adotar na transposição para a praxis. À medida que estas pontes são solidificadas, o processo torna-se mais ágil, mais eficaz e mais eficiente. Assim, da definição de um problema clínico à realização da sistematização do conhecimento (revisões sistemáticas), à avaliação crítica dos mesmos, à tomada de decisão relativamente aos achados e à implementação de medidas (protocolos de procedimentos, processos de auditoria, Guidelines, algoritmos de decisão, fluxogramas, entre outros) (Galvão, Sawada, \& Trevizan, 2004), todas as etapas são importantes para garantir o uso do conhecimento na clínica.

Para além dos produtos criados, as reuniões e a formação das diferentes equipas possibilitam a transposição dos resultados, sobretudo pela aposta na reflexão prévia sobre o método adotado e sobre a melhor estratégia de implementação que promova a mudança de comportamento (Cunha, Rego, Cunha, Cabral-Cardoso, \& Neves, 2016).

Para conduzir um processo de mudança eficaz, a aposta tem sido na mudança de atitude e na educação/formação dos intervenientes, analisando a situação em três planos: o tipo de foco/problema; as causas do problema e o tempo disponível para executar a mudança (Cunha et al., 2016).

De salientar que os protocolos, os fluxogramas e os algoritmos de decisão clínica potenciam a eficácia de todo o processo, por agilizar a mudança. Corroboramos a opinião de Cunha et al. (2016) ao advogar que quando o tempo disponível para a implementação é curto, a criação de normas de orientação clínica, procedimentos de atuação e Guidelines orientadores, são estratégias eficazes, envolvendo o menor número de intervenientes na planificação da mudança, com indicações claras e precisas, e com necessidade à gestão dos fatores de resistência à mudança.

As normas de orientação clínica são plataformas de normalização dos cuidados de saúde, alinhando as práticas dos profissionais de acordo com as recomendações das sociedades profissionais, e não pela experiência empírica do quotidiano, assegurando a qualidade, segurança, confiabilidade e eficiência dos serviços (Santos, Azevedo, \& Vaz-Carneiro, 2015). 
A monitorização é crucial no processo de implementação, identificando acontecimentos imprevistos que possam funcionar como oportunidades ou ameaças ao processo (resistências à mudança). As auditorias como processo de monitorização e avaliação contínua, favorecem a implementação da mudança, através da supervisão e correção.

Em suma, a resolução eficaz do problema irá depender essencialmente do rigor científico adotado na análise dos dados, bem como da estratégia adotada na sua implementação na práxis.

\section{Diretrizes para outros estudos}

O trabalho colaborativo desenvolvido aponta soluções para a introdução da evidência na clínica, com os profissionais a reconhecer a PBE como uma competência para a tomada decisão baseada na melhor evidência disponível para o cuidado com o cliente (Baixinho \& Ferreira, 2019). Estudos futuros devem explorar a tomada de decisão clínica, suportada pela evidência e como o conhecimento empírico e o conhecimento intuitivo podem influenciar a PBE.

Por outro lado, os resultados sobre o envolvimento dos estudantes da formação prégraduada, são animadores e justificam não só a continuidade do seu envolvimento, como também a necessidade de aprofundar os benefícios no aumento da literacia científica e na utilização da investigação no início da sua atividade profissional, comparando com os recém-licenciados que não tiveram oportunidades similares.

Concordamos com Melnik, Souza e Carvalho (2014) ao sustentar que, para o avanço de uma PBE seja importante, identificar e esclarecer equívocos associados à leitura e compreensão das publicações científicas bem como, promover a comunicação entre os pesquisadores e clínicos. Esta é uma área nobre para investigações futuras poderem explorar processos e produtos para a difusão do conhecimento. É imperioso identificar a fonte da evidência científica (estudos primários ou secundários), demonstrar a avaliação da qualidade, quer a nível da sua robustez (validade e fiabilidade) quer da sua relevância e aplicabilidade para o contexto local (Sousa, Marques-Vieira, Severino, \& Antunes, 2017) e transformá-la num produto aplicável na clínica.

Importa ainda incentivar o consumo da melhor evidência, bem como desenvolver pesquisas com o envolvimento de todos os atores do processo, que permitam a produção de conhecimento no seu contexto natural, com contributo para a qualidade de vida/ganhos em saúde e satisfação dos profissionais.

\section{Aplicabilidade em outros contextos}

Este painel de discussão apresenta a experiência dos investigadores no projeto Transição Segura, no entanto a questão central - a transferência do conhecimento para a clínica - é transversal a todos os contextos de prestação de cuidados de saúde. Os investigadores e profissionais de outras áreas são bem-vindos ao painel porque esta problemática não é exclusiva das ciências da saúde e a visão transdisciplinar pode enriquecer o painel e a discussão. Os passos para a TC incluem o compromisso organizacional, a identificação de prioridades para a qualidade e segurança dos cuidados de saúde, a criação e/ou desenvolvimento de redes para o desenvolvimento do conhecimento e sua introdução na clínica, criação de linhas de investigação amplas e em sinergia com os objetivos operacionais do serviço, definição de objetivos a curto e médio prazo e respetiva identificação dos indicadores de avaliação de processo e resultado, orçamentar os ganhos com a TC e a validação dos resultados (intermédios e finais) da investigação com os clínicos. A metodologia apresentada tem potencialidade para ser utilizada por outros investigadores, em contextos diferentes. 
A PBE e a transferência do conhecimento são temas atuais nos debates em saúde.

A experiência dos participantes no projeto transição segura e os resultados do mesmo em confronto com as experiências internacionais, bem como a discussão trazida pelos participantes fornecerão algumas ferramentas para o planeamento da TC.

\section{Conclusões}

O desenvolvimento da investigação e a evolução das ciências da saúde e tecnologias subjacentes, fez emergir um aumento exponencial de estudos, todavia com uma baixa representatividade na melhoria das práticas clínicas e na gestão das políticas de saúde. A demora para tal efetivação parece assentar na adequação do desenho de estudo à práxis, nos obstáculos à sua disseminação, bem como o perfil investigativo de algumas instituições ou na própria formação pré-graduada nos cursos da área da saúde.

A metodologia qualitativa permite uma expressão social, traduzindo como os processos são experienciados e pondo a descoberto as interpretações que lhe são imputadas. O conhecimento compreensivo emergente, permite o desenvolvimento da teoria, descrição, explicação enfatizando a interpretação como característica central da investigação. A complexidade dos fenómenos encontra assim uma forma de se transformar em evidência científica através de métodos e técnicas, intercâmbio e aplicação do conhecimento.

O projeto Transição Segura, sustentado na resolução de problemas e na transferência do conhecimento para a clínica, tem possibilitado o desenvolvimento das competências dos profissionais, destacando-se a produção e a divulgação do conhecimento, e consequente melhoria dos cuidados de saúde. Esta aquisição de competências científicas tem refletido um conjunto mínimo essencial de atributos, conhecimentos, habilidades e atitudes, permitindo ao profissional realizar atividades baseadas na evidência. Torna-se imperativo modelos interativos e produtivos que promovam o aprender a investigar e que possam atuar não só ao nível individual, mas também organizacional das políticas de saúde, garantindo que, desde o início, a pesquisa é projetada para estar atenta às necessidades da prática.

\section{Referências}

Albarqouni, L., Hoffmann, T., Straus, S., et al. (2018). Core Competencies in Evidence-Based Practice for Health Professionals: Consensus Statement Based on a Systematic Review and Delphi Survey. JAMA Netw Open, 1(2), e180281. doi: 10.1001/jamanetworkopen.2018.0281

Atkins, S., Lewin, S., Smith, H., Engel, M., Fretheim, A., \& Volmink, J. (2008). Conducting a metaethnography of qualitative literature: Lessons learnt. BMC Medical Research Methodology, 8. doi: https://doi.org/10.1186/1471-2288-8-21

Baixinho, C. L., Ferreira, Ó., Marques, F. M., Presado, M. H., \& Cardoso, M. (2017). Transição segura: um projeto da translação do conhecimento para a prática clínica. In A. P. Costa, M.C Sánches-Gómez, \& M. V. M, Cilleros (Eds.). A prática na Investigação Qualitativa: exemplos de estudos (pp.57-80). Oliveira de Azeméis: Ludomedia.

Baixinho, C. L., Ferreira, Ó., Marques, F. M., Presado, M. H., Cardoso, M., \& Sousa, A. D. (2018). Investigação qualitativa e transferência do conhecimento para a clínica. In C. Brandão, J. L. Carvalho, J. Ribeiro, \& A. P. Costa (Eds.). A prática na Investigação Qualitativa: exemplos de estudos (vol. 2) (pp.179-208). Oliveira de Azeméis: Ludomedia.

Baixinho, C. L., Ferreira, Ó., Marques, F. M., Presado, M. H., Cardoso, M., \& Sousa, A. D. (2019). Transferir conhecimento para a clínica - passo-a-passo: resultados do projeto transição segura. In C. Brandão, J. L. Carvalho, R. Arellano, C.L.Baixinho, \& J. Ribeiro (Eds.). A prática na Investigação Qualitativa: exemplos de estudos (vol. 3) (pp.87-104). Oliveira de Azeméis: Ludomedia. 
Baixinho, C.L., \& Ferreira, Ó. (2019). Aprender em projetos de translação de conhecimento durante o ensino clínico. In A. P. Costa, A. A. Sá, P. Castro, D. N. Souza (Eds.). Atas do $8^{o}$ Congresso Ibero-Americano em Investigação Qualitativa. Oliveira de Azeméis: Ludomedia, pp.159-68.

Baumbusch, J. L., Kirkham, S. R., Khan, K. B., McDonald, H., Semeniuk, P., Tan, E., \& Anderson, J. M. (2008). Pursuing common agendas: a collaborative model for knowledge translation between research and practice in clínical settings. Res Nurs Health, 31(2), 130-40. doi: 10.1002/nur.20242

Broeiro, P. (2015). Prática baseada em evidência e seus limites. Rev Port Med Geral Fam.,31, 238-40.

Canadian Institutes of Health Research. (2014). More about knowledge translation at CIHR: knowledge translation definition. Available from: http://www.cihr-irsc.gc.ca/e/39033.html

Choi, A. R., Choi, A. R., Cheng, D. L., \& Greenberg, P. B. (2019). Twelve tips for medical students to conduct a systematic review. Medic Teach, 41(4), 471-5. Epub 2018 Jan 23. doi: 10.1080/0142159X.2018.1426847

Cochrane. (2018). What is a systematic review? Cochrane Consumer Network. Available from: https://consumers.cochrane.org/what-systematic-review

Cunha, A., Rego, A., Cunha, R., Cardoso, C., \& Neves, P. (2016). Manual de Comportamento Organizacional e Gestão ( $8^{\underline{a}}$ edição). Lisboa: Editora $\mathrm{RH}$.

Donato, H., \& Donato, M. (2019). Etapas na Condução de uma Revisão Sistemática. Acta Med Port 2019, 32(3), 227-235. https://doi.org/10.20344/amp.11923

Galvão, C., Sawada, N., \& Trevizan, M. (2004). Revisão sistemática: recurso que proporciona a incorporação das evidências na prática da enfermagem. Rev Lat. Am. Enferm., (12)3, 54956. doi: https://doi.org/10.1590/S0104-11692004000300014

Hestevik, C. H., Molin, M., Debesay, J., Bergland, A., \& Bye, A. (2019). Older persons' experiences of adapting to daily life at home after hospital discharge: A qualitative metasummary. BMC Health Services Research, 19(1), 1-13. https://doi.org/10.1186/s12913-019-4035-z

Lachal, J., Revah-Levy, A., Orri, M., \& Moro, M. R. (2017). Metasynthesis: An Original Method to Synthesize Qualitative Literature in Psychiatry. Frontiers in Psychiatry, 8(269). https://doi.org/10.3389/fpsyt.2017.00269

Lage, I., Vilaça, S., Araújo, O., Almendra, M., Novais, R., \& Braga, F. (2016). Prática Baseada na Evidência em Enfermagem: estado da arte. In P. Membiele, N. Cascado \& M. I. Cabreiros (org.s). Nuevos escenarios en la docência universitária/Novos escenarios na docência universitária. Roma: Edita Educación Editora.

Lopes, A. L. M., \& Fracolli, L. A. (2008). Revisão sistemática de literatura e metassíntese qualitativa: considerações sobre sua aplicação na pesquisa em enfermagem. Texto Contexto Enferm, 17(4), 771-8. https://doi.org/10.1590/s0104-07072008000400020

Martsolf, D. S., Draucker, C. B., Cook, C. B., Ross, R., Stidham, A. W., \& Mweemba, P. (2010). A meta-summary of qualitative findings about professional services for survivors of sexual violence. Qual Rep, 15(3), 489-506. doi: PMID: 21837284

Matheus, M.C.L. (2009). Metassíntese qualitativa: desenvolvimento e contribuições para a prática baseada em evidências. Acta Paulista de Enfermagem, 22(spe1), 5435. https://doi.org/10.1590/S0103-21002009000800019

Mena-Tudela, D., González-Chordá, V. M., Cervera-Gasch, A., Maciá-Soler, M. L., \& Orts-Cortés, M.I. (2018). Effectiveness of an Evidence-Based Practice educational intervention with second-year nursing students. Revista Latino-Americana de Enfermagem, 26, e3026. Epub August 09, 2018. https://doi.org/10.1590/1518-8345.2502.3026

Melnik, T., Souza, W.F., \& Carvalho, M.R. (2014). A importância da prática da psicologia baseada em evidências: aspectos conceituais, níveis de evidência, mitos e resistências. Revista Costarricense de Psicología, 33(2), 79-92.

Mendes, K.D.S., Silveira, R.C.C.P., \& Galvão, C.M. (2008). Revisão integrativa: método de pesquisa para a incorporação de evidências na saúde e na enfermagem. Texto Contexto Enfermagem, 17(4), 758-64. http://dx.doi.org/10.1590/S0104-07072008000400018

$$
\text { - } 101 \text { - }
$$


Pearson, A., Jordan, Z., \& Munn, Z. (2012). Translational science and evidence-based healthcare: a clarification and reconceptualization of how knowledge is generated and used in healthcare. Nurs Res Pract., 792519. Epub 2012 Feb 14. doi: 10.1155/2012/792519

Pereira, E.R. (2013). Translation of knowledge and translational research in healthcare. J Nurs UFPE on line, 7(3), 1-3. doi: 10.5205/01012007

Sandelowski, M., Barroso, J., \& Voils, C. I. (2007). Using qualitative metasummary to synthesize qualitative and quantitative descriptive findings. Res Nurs Health, 30(1), 99-111. doi: https://doi.org/10.1002/nur.20176

Santos, G., Azevedo, P., \& Vaz-Carneiro, A. (2015). Normas de Orientação Clínica 2.0. Act Med Port., 28(3), 395-414.

Sousa, L.M.M., Marques-Vieira, C.M.A., Severino, S.S.P., \& Antunes, A.V. (2017). A metodologia de revisão integrativa da literatura em enfermagem. Revista Investigação em Enfermagem, Ser.II(21), 17-26.

Urquhart, C. (2010). Systematic reviewing, meta-analysis and meta-synthesis for evidence-based library and information science. Information Research, 15(3), 13-5.

\section{Notas biográficas}

Cristina Lavareda Baixinho. Professora-Adjunta na Escola Superior de Enfermagem de Lisboa Doutora em Enfermagem. Mestre em Saúde Escolar. Especialista em Enfermagem de Reabilitação. Investigadora no ciTEchCare, Leiria, Portugal. Áreas de Investigação: Gestão do Risco de Queda em idosos residentes em estruturas residenciais para idosos e na comunidade; transição do hospital para a comunidade; Prática Simulada; Prevenção de Lesões músculo-esqueléticas nos enfermeiros especialistas. Editora da Anna Nery Revista de Enfermagem. Membro da Comissão Científica do CIAIQ 2020.

Óscar Ferreira. Professor Adjunto na Escola Superior de Enfermagem de Lisboa. Doutor em Educação. Mestre em Educação Médica. Licenciado em Administração dos Serviços de Enfermagem. Especialista em Enfermagem Médico-Cirúrgica. Ex-Vice-Presidente da Direção da Escola Superior de Enfermagem Francisco Gentil (2006/2007). Investigador da Linha de História de Enfermagem da UI\&DE. Colaborador estrangeiro do Laboratório de História do Cuidado e Imagem em Enfermagem - Lacuiden da Universidade Federal do Rio de Janeiro. Presidente da Direção da Associação Nacional de História de Enfermagem (ANHE). Secretário da Assembleia Geral da Associação da História de Educação de Portugal (HISTEDUP). Membro da Sociedade Portuguesa de Ciências da Educação (SPCE).

Fátima Mendes Marques. Professora-Adjunta na Escola Superior de Enfermagem de Lisboa. Doutora em Educação. Mestre em Ciências de Educação. Especialista em Enfermagem de Reabilitação. Investigadora no CeiED (Centro de Estudos Interdisciplinares em Educação e Desenvolvimento) e UI\&DE (Unidade de Investigação \& Desenvolvimento em Enfermagem). Áreas de Investigação: Tomada de decisão em Enfermagem, Educação em Enfermagem, Prevenção de Lesões músculo-esqueléticas nos Enfermeiros especialistas. Membro da Comissão Científica do CIAIQ 2020.

Maria Helena Presado. Professora-Adjunta na Escola Superior de Enfermagem de Lisboa. Doutora em Psicologia ramo de Psicologia Clínica e da Saúde. Mestre em Comportamento Organizacional. Especialista em Enfermagem de Saúde Materna e Obstetrícia. Investigadora na CEMRI (Centro de Estudos Multiculturais da Universidade Aberta) e UI\&DE. Áreas de Investigação: Transição para a menopausa, Saúde da Mulher; Prática Simulada; Prevenção de Lesões músculo-esqueléticas nos Enfermeiros especialistas. Coordenadora do Mestrado em Enfermagem de Saúde Materna e Obstetrícia da ESEL (2015-2018). Membro da Comissão Científica do CIAIQ 2020.

Mário Cardoso. Professor Adjunto na Escola Superior de Enfermagem de Lisboa. Doutorando em Ciências da Educação da Universidade Lusófona de Humanidades e Tecnologia de Lisboa. Curso de Especialização em Ciências da Educação na Universidade Lusófona de Humanidades e Tecnologia de Lisboa. Título de Especialista da Escola Superior de Enfermagem de Lisboa. Especialidade em Enfermagem de Saúde Materna e Obstetrícia. Áreas de Investigação: Transição para a Menopausa, Saúde da Mulher; Prática Simulada; Prevenção de Lesões músculo-esqueléticas nos Enfermeiros especialistas. 
Armando David. Enfermeiro no Centro Hospitalar do Funchal. Mestrando em Enfermagem de Saúde Materna e Obstetrícia pela Escola Superior de Enfermagem de Lisboa. Mestrando em Gestão em Enfermagem pela Escola Superior de Enfermagem de Lisboa. Título de Especialista na área das Ciências de Enfermagem pela Escola Superior de Enfermagem São Francisco das Misericórdias. Especialista em Enfermagem de Saúde Materna e Obstetrícia. Áreas de Investigação: Prevenção de Lesões músculo-esqueléticas nos Enfermeiros especialistas e Prática Simulada.

Tiago Nascimento. Professor Assistente Convidado na Escola Superior de Enfermagem de Lisboa. Enfermeiro na Santa Casa da Misericórdia de Lisboa. Mestre em Enfermagem na Área de Especialização de Gestão em Enfermagem. Mestre em Enfermagem na Área de Especialização de Enfermagem Comunitária. Doutorando em Gestão na Universidade Europeia. Membro da Associação de Enfermagem em Cuidados Continuados e Paliativos. Áreas de Investigação: sistemas de informação; modelos de organização de cuidados; stress nas organizações; saúde da mulher. 